Review began 11/26/2021 Review ended 11/27/2021 Published 11/28/2021

๑) Copyright 2021

Alvarez-Payares et al. This is an open access article distributed under the terms of the Creative Commons Attribution License CC-BY 4.0., which permits unrestricted use, distribution, and reproduction in any medium, provided the original author and source are credited.

\section{Unexplained Anemia in the Elderly}

Jose C. Alvarez-Payares ${ }^{1}$, Sebastián Rivera-Arismendy ${ }^{1}$, Pablo Ruiz-Bravo ${ }^{2}$, Sara M. Sánchez-Salazar ${ }^{2}$, Rene A. Manzur ${ }^{1}$, Sara I. Ramirez-Urrea ${ }^{3}$, Andres Puello ${ }^{4}$

1. Internal Medicine, University of Antioquia, Medellín, COL 2. Internal Medicine, University of Antioquía, Medellín, COL 3. General Medicine, Fundación Universitaria San Martín, Medellín, COL 4. Internal Medicine, University of Sucre, Sincelejo, COL

Corresponding author: Jose C. Alvarez-Payares, josecarlosalvarezj9@hotmail.com

\begin{abstract}
Anemia is frequently diagnosed in elderly patients, and it is a key indicator of many reactive and clonal conditions. Furthermore, the older age is the most common presenting age for myelodysplastic syndromes (MDS). Anemia in older age may be attributed to an inflammatory state due to senescence, comorbidities, nutritional deficiencies, or primary bone marrow conditions. As diagnostic possibilities and life expectancy increase, the prevalence of anemia of the elderly increases as well. The etiology has a direct impact on the treatment and quality of life of these patients, in whom is a usual clinical challenge as it may be due to a multifactorial origin. In a minority group, when no etiology is identified, it is classified as unexplained anemia (UA) or clonal cytopenia of unknown significance (CCUS). The underlying cause of anemia remains unexplained in $30 \%$ of cases, and a great part of unexplained cytopenia may account for myeloid neoplasms. Anemia in the elderly is associated with worse cognitive and functional outcomes and increased mortality.
\end{abstract}

Categories: Internal Medicine, Hematology

Keywords: clonal cytopenia, myelodysplastic syndromes, chronic inflammatory diseases, elderly patient, anemia

\section{Introduction And Background}

Anemia is a common condition in elderly age, with a prevalence up to $17 \%$ in patients older than 65 years [1]; in addition, its prevalence has increased with higher life expectancy and better diagnostic tests. In older patients, it is more frequently associated with gastrointestinal (GI) diseases, chronic kidney disease (CKD), cancer, and myelodysplastic syndrome (MDS). Due to elder age and its own setting, different causes may add up and promote anemia development, generating a diagnostic and treatment challenge [2].

According to its etiology, anemias may be classified as nutritional deficiency anemia, bleeding anemia, clonal anemia, and secondary to chronic inflammatory diseases (including CKD).

Identifying the cause is paramount to offer a specific and efficient therapy for this population. When no cause is identified, patients were previously classified to have unexplained anemia (UA), as was described by Artz et al. [3]. Nonetheless, recent classifications based on bone marrow (BM) studies suggest that it must be classified as idiopathic cytopenia of unknown significance (ICUS) with isolated anemia (ICUS-A) [4]. Furthermore, a condition known as clonal cytopenia of unknown significance (CCUS) has been described, which consists of cytopenic patients with somatic mutations in peripheral blood leukocytes that do not meet diagnostic criteria for MDS or other neoplastic BM conditions [4].

In patients with unknown etiology or numerous comorbidities, anemia management requires a multidisciplinary approach that grants the understanding of the physiologic changes of elderly age. In most cases, nutritional supplementation or etiology removal would be enough; however, long-term treatment with directed therapy, continuous erythropoietin (EPO) therapy, or blood transfusions may be required. The main goal is a clear impact on the quality of life and safety of the patient [2]. In the present article, we review current concepts surrounding clinical relevance, pathogenesis, and management of UA on elderly patients.

\section{Review}

\section{Definition of anemia related to older age}

Although hemoglobin levels decrease with age, we will assume the definition of anemia in accordance with the World Health Organization (WHO) criteria, which was defined as a hemoglobin concentration of $<13.0$ $\mathrm{g} / \mathrm{dL}$ in men and $<12.0 \mathrm{~g} / \mathrm{dL}$ in women [5].

\section{Epidemiology of anemia on elderly patients}

Large prospective studies have revealed a general prevalence of anemia of $10 \%-24 \%$ in older adults [5]. Anemia is one of the main conditions of elderly inpatients, with a prevalence of $40 \%$, being most common in nursing homes with $47 \%$. Prevalence increases with age, up to $50 \%$ in men older than 80 years, both 


\section{Cureus}

inpatient and outpatient (Figure 1). An increase of such figures is expected due to longer aging associated with more diagnostic possibilities [5].

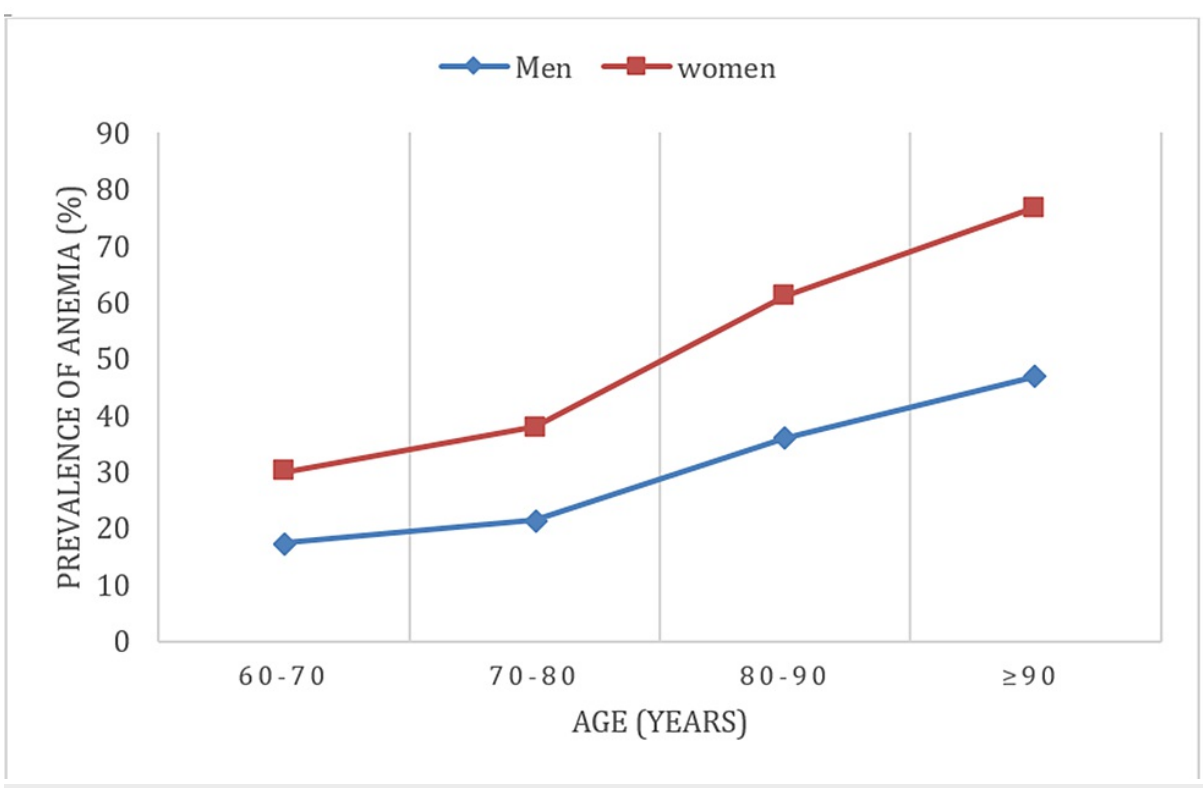

FIGURE 1: Increase of anemia prevalence in older adults

Increase of anemia prevalence in patients with advanced age from a cohort of 19758 inpatient and outpatient visits from university hospitals (modified from Steensma et al. [6])

\section{Clinical relevance of older age anemia}

Anemia is associated with a wide spectrum of clinically relevant conditions; hence, identifying even mild anemia is crucial (Table 1). Low hemoglobin levels represent a risk factor for cardiovascular diseases [7], cognitive impairment [8], insomnia [9], mood disorders [10], worse quality of life [11,12], and impaired executive function and physical performance [11,13], with higher fall and fracture risk [12].

Anemia presence is associated with frequent hospital admissions [14] and longer inpatient stance [15]; therefore, it has been concluded that anemia is a mortality marker [16], with higher mortality risks when anemia is secondary to nutritional disorders or CKD; both may be observed in patients with UA. 


\section{Cureus}

\begin{tabular}{lllll}
\hline Etiology & Frequency (\%) & & \\
Country & USA-1 [2] & USA-2 [17] & Italy [18] & Poland [9] \\
Iron deficiency & 16.6 & 25.3 & 16.0 & 13.0 \\
B12- and/or folate-deficiency anemia & 14.3 & $<1$ & 9.5 & 7.1 \\
Iron- and B12- and/or folate-deficiency anemia & 3.4 & - & - & 2.4 \\
Chronic disease/inflammation anemia & 19.7 & 9.8 & 17.4 & 33.1 \\
CKD anemia & 8.2 & 3.4 & 15.0 & 1.2 \\
CKD and chronic disease/inflammation anemia & 4.3 & - & - & - \\
UAE & 33.6 & 43.7 & 26.4 & 28.4 \\
Clonal hematopoiesis & - & 7.5 & 1.8 & - \\
Others & - & 10.3 & 14.4 & 14.8 \\
\hline
\end{tabular}

TABLE 1: Distribution of anemia etiology in elderly patients (data from four studies[2,9,17,18]) CKD: chronic kidney disease; UAE: unexplained anemia of the elderly

\section{Pathogenesis and basic mechanisms of anemia in elderly age}

Anemia in the elderly usually has multiple underlying causes and is frequently associated with more than one predisposing factor. Based on physiologic concepts, underlying diseases may be classified as inflammatory anemia (especially due to CKD), nutritional deficiency, and clonal hematopoiesis. Insufficient EPO production is remarkable, which is commonly observed in patients with CKD; however, it may be observed in patients with normal renal function, which is a key factor in patients with clonal cytopenia of unknown significance [6]. Patients with underlying inflammatory disorders may present an increased hepcidin hepatic production, which leads to decreased erythropoiesis and iron uptake in the reticuloendothelial system. Furthermore, EPO production is insufficient in response to anemia, with a mild impact of EPO on erythropoiesis (Figure 2). Another distinctive characteristic is increased phagocytosis of senescent red blood cells (eryptosis).

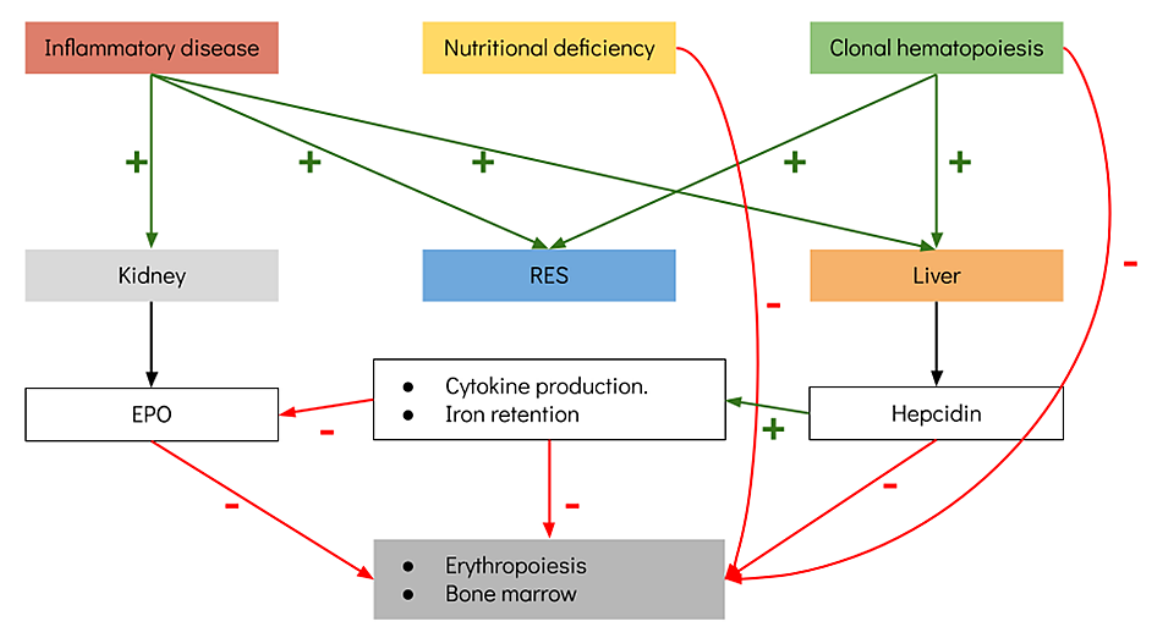

\section{FIGURE 2: Possible mechanisms of anemia in elderly adults}

(Modified from Shlush [19])

In the elderly patient with anemia, an initial workup to rule out GI bleeding and nutritional deficiency of common factors that could lead to anemia (B12, folate, iron) is mandatory. After this initial workup, it is essential to remember that one-third of patients with CKD or inflammatory anemia (related to cancer, 


\section{Cureus}

autoimmune disease, or chronic infections) may present a pro-inflammatory state, with insufficient EPO production. This is directly related to increased hepcidin levels, an important acute-phase protein synthesized in the liver. Hepcidin presence leads to a reduction of iron intestinal absorption and reduced iron release by macrophages [19-21]. Patients with cancer, CKD, and autoimmune diseases and elderly adults may all have elevated hepcidin levels, attributed to a pro-inflammatory state or "inflamm-aging," which is directly related to age. Many pro-inflammatory cytokines are increased in this state, such as IL-1, IL-6, and tumor necrosis factor-alpha (TNFa); conversely, a reduction of autophagy secondary to increased NF- $\mathrm{kB}$ signaling and increased reactive oxygen species (ROS) may lead to increased inflammasome response [20].

In such a pro-inflammatory state, it is paramount to identify whether or not it is due to physiologic changes related to aging or if it is evidence of systemic response of an unidentified condition. Slightly elevated IL-6 levels, which are caused by changes of body composition or underlying inflammatory disease, may inhibit EPO production and/or hepcidin activation. Difficulties in IL-6 detection limit the capabilities of routine assessment [20].

In elderly adults, clonal leukocytes have been detected, with somatic mutations increasing with age. This clonal hematopoiesis is related to a higher prevalence/mortality of hematologic malignancies such as MDS $[22,23]$. In healthy subjects without cytopenias, this condition is called clonal hematopoiesis of indeterminate potential (CHIP); however, once there is anemia, the diagnosis changes to clonal cytopenia of unknown significance (CCUS) or MDS, considering that many patients with CCUS progress to MDS (Table 2) $[22,23]$. Both CHIP and CCUS patients may develop other hematologic malignancies; hence, a leukocyte thorough study is recommended to establish whether or not somatic mutations are present in patients with unexplained cytopenias, as well as bone marrow plus flow cytometry evaluation to determine if cytogenetic anomalies are present [24].

\begin{tabular}{|c|c|c|c|c|c|}
\hline Condition & UA & ICUS & CCUS & CHIP & MDS \\
\hline Cytopenia & + & + & + & - & + \\
\hline Dysplasıa & nd & - & - & - & + \\
\hline Cytogenetıc abnormalities & nd & - & - & - & + \\
\hline Somatic mutations & nd & - & + & + & + \\
\hline
\end{tabular}

\section{TABLE 2: Classification of aging-related clonal hematopoiesis}

UA: unexplained anemia; ICUS: idiopathic cytopenia of undetermined significance; CCUS: clonal cytopenia of undetermined significance; CHIP: clonal hematopoiesis of indeterminate potential; MDS: myelodysplastic syndrome; nd: not detected

Patients with cytopenia without molecular abnormalities nor criteria for other diseases such as MDS will be included in the idiopathic cytopenia of unknown significance (ICUS) group and those with anemia in the idiopathic cytopenia of unknown significance with anemia (ICUS-A) group [24]. As ICUS-A prevalence is directly related to age, this has been considered as "anemia of older age" [25].

A reduction in EPO levels in the absence of CKD or any other inflammatory causes would lead to a possible ICUS-A, which could be explained by an intrinsic defect of EPO in the presence of an older kidney or a lower testosterone and estrogen production. It is still unclear whether it is possible or not for ICUS-A to present with an adequate EPO production [26]. Patients with MDS may also present with lower EPO production, which may respond to recombinant EPO treatment [26].

\section{Diagnostic aspects}

The basic anemia workup in elderly patients requires that the most common etiologies be ruled out through different diagnostic studies (Tables 3, 4). Each patient's context will guide the need for additional studies such as upper gastrointestinal tract endoscopy or abdominal ultrasound. In the presence of additional anomalies or signs of clonal hematologic disease, a bone marrow biopsy and aspiration is mandatory to rule out hematologic diseases, including MDS [2]. As with many other conditions, anemia workup must be directed by the patient's life expectancy; many authors consider that a bone marrow aspiration is adequate when life expectancy is at least three months [27]. 


\section{Cureus}

Initial tests
Hematology
Blood chemistry
Serum iron parameters
Nutritional anemia parameters
Secondary tests
Hemolysis suspicion
Other tests in men
Other negative tests with macrocytosis and/or other
cytopenias
Isolated normocytic anemia with a reticulocyte count
of $10000 / \mu L$ or less

Tests

Complete blood count

Creatinine, blood urea nitrogen, glomerular filtration rate creatinine

Iron, ferritin, total iron-binding capacity

Serum B12, red blood cell folate levels

Tests

Bilirubins, lactate dehydrogenase, reticulocyte count, direct antiglobulin test, peripheral blood smear, haptoglobin

Serum testosterone

Bone marrow examination or molecular profiling for clonal hematopoiesis

Bone marrow examination for pure red cell aplasia

TABLE 3: Laboratory tests on the elderly patient

\begin{tabular}{|c|c|c|c|c|c|c|c|c|}
\hline $\begin{array}{l}\text { Type of } \\
\text { anemia }\end{array}$ & MCV & Iron/TIBC & Ferritin & ESR/CRP & EPO & $\mathrm{CrCl}$ & Albumin & Miscellaneous \\
\hline IDA & Small & Low/high & Low & $\mathrm{nl}$ & High & $\mathrm{nl}$ & $\mathrm{nl}$ & \\
\hline UA/CDA & Small & Low/high & Low/high & Alto & $\mathrm{BI}$ & $\mathrm{nl}$ & Low-nl & \\
\hline CKD & $\mathrm{nl}$ & $\mathrm{nl}$ & $\mathrm{nl}$ & $\mathrm{nl}$ & Low & $<30 \mathrm{~mL} /$ minute & Low-nl & \\
\hline Hypothyroidism & Large & $\mathrm{nl}$ & $\mathrm{nl}$ & $\mathrm{nl}$ & High & $\mathrm{nl}$ & $\mathrm{nl}$ & High TSH \\
\hline B12/folate & Large & $\mathrm{nl}$ & $\mathrm{nl}$ & $\mathrm{nl}$ & High & $\mathrm{nl}$ & $\mathrm{nl}$ & Low vitamin levels, high MMA \\
\hline MDS & Large & $\mathrm{nl}$ & $\mathrm{nl}$ & $\mathrm{nl}$ & High & $\mathrm{nl}$ & $\mathrm{nl}$ & Bone marrow study may be diagnostic \\
\hline Desnutrition & $\mathrm{nl}$ & $\mathrm{nl}$ & $\mathrm{nl}$ & $\mathrm{nl}$ & $\mathrm{Bl}$ & $\mathrm{nl}$ & Low & \\
\hline UA & $\mathrm{nl}$ & $\mathrm{nl}$ & $\mathrm{nl}$ & $\mathrm{nl}$ & $\mathrm{Bl}$ & $\mathrm{nl}$ & $\mathrm{nl}$ & \\
\hline
\end{tabular}

\section{TABLE 4: Laboratory findings of common anemias in elderly adults}

IDA: iron-deficiency anemia; UA: unexplained anemia; CDA: chronic disease anemia; CKD: chronic kidney disease; MDS: myelodysplastic syndrome MCV: mean corpuscular volume; TIBC: total iron-binding capacity; ESR: erythrocyte sedimentation rate; CRP: C-reactive protein; EPO: erythropoietin; $\mathrm{CrCl}$ : creatinine clearance; TSH: thyroid-stimulating hormone; MMA: methylmalonic acid

It is correct to assume that a patient may have a bone marrow clonal disorder if no concluding results are available after a molecular evaluation, cytogenetic studies, and/or flow cytometry are performed. The detection of clonal myeloid cells may cause a change in the diagnosis, i.e., from ICUS to CCUS or even MDS [27].

\section{Management}

No reports have been published describing the natural history of UA or if treatment may improve any of the adverse associations previously described. In UA, therapy is not usually indicated [28]. Anemia degree is usually modest; a severe/symptomatic anemia may lead to a diagnosis reassessment.

A study has demonstrated an improved hemoglobin concentration and quality of life in African-American elderly patients with chronic UA previously treated with EPO [17]; however, no proper studies have been designed to evaluate EPO therapy for UA patients. 
A significant proportion of male patients with UA and low testosterone levels will improve their hemoglobin concentration after testosterone therapy [18]. Accordingly, a multicentric, randomized clinical trial was published, which evaluated testosterone treatment in elderly male patients with low testosterone levels at baseline [18]. Testosterone gel was administered with adjusted doses to maintain testosterone levels in a normal range for 12 months. The results showed that, from 788 patients, 126 were anemic and 62 were classified as having UA. More than 50\% of anemic subjects had a hemoglobin increase of $>1 \mathrm{~g} / \mathrm{dL}$ at month 12 of treatment. Despite this encouraging result, investigators properly noted that no health benefit has been observed from a higher hemoglobin level [18].

Some patients with UA and normal ferritin (but lower than $200 \mathrm{ng} / \mathrm{mL}$ ) may respond to intravenous iron [29]. In these patients, a previously undiagnosed iron-deficiency anemia must be assumed, and an extensive workup for a bleeding source is mandatory [29].

At the moment, different drugs with hypoxia-inducible factors, which may impact patients with low endogenous EPO levels, represent a future treatment option for UA in elderly patients [27].

\section{Conclusions}

Anemia in older persons is a challenge in clinical practice. In many cases, various etiologies could be detected, and a meticulous investigation leads to the correct diagnosis. In a great proportion of patients, no underlying cause of anemia is found after the first evaluation, resulting in the provisional diagnosis of UA. Nevertheless, in plenty of cases, no underlying etiology is found even after a thorough diagnostic workup that includes an examination of different organ systems, including the bone marrow, and cytogenetic and molecular studies. In these kinds of patients, in many cases, only follow-up can be offered. Finally, it is difficult to determine which patients with unexplained anemia could closely develop hematological neoplasms, although at present there are already great advances in this regard.

\section{Additional Information \\ Disclosures}

Conflicts of interest: In compliance with the ICMJE uniform disclosure form, all authors declare the following: Payment/services info: All authors have declared that no financial support was received from any organization for the submitted work. Financial relationships: All authors have declared that they have no financial relationships at present or within the previous three years with any organizations that might have an interest in the submitted work. Other relationships: All authors have declared that there are no other relationships or activities that could appear to have influenced the submitted work.

\section{References}

1. Gaskell H, Derry S, Andrew Moore R, McQuay HJ: Prevalence of anaemia in older persons: systematic review. BMC Geriatr. 2008, 8:1. 10.1186/1471-2318-8-1

2. Goodnough LT, Schrier SL: Evaluation and management of anemia in the elderly . Am J Hematol. 2014, 89:88-96. 10.1002/ajh.23598

3. Artz AS, Thirman MJ: Unexplained anemia predominates despite an intensive evaluation in a racially diverse cohort of older adults from a referral anemia clinic. J Gerontol A Biol Sci Med Sci. 2011, 66:925-32. 10.1093/gerona/glr090

4. Valent P, Orazi A, Steensma DP, et al.: Proposed minimal diagnostic criteria for myelodysplastic syndromes (MDS) and potential pre-MDS conditions. Oncotarget. 2017, 8:73483-500. 10.18632/oncotarget.19008

5. Stauder R, Thein SL: Anemia in the elderly: clinical implications and new therapeutic concepts . Haematologica. 2014, 99:1127-30. 10.3324/haematol.2014.109967

6. Steensma DP, Tefferi A: Anemia in the elderly: how should we define it, when does it matter, and what can be done?. Mayo Clin Proc. 2007, 82:958-66. 10.4065/82.8.958

7. Culleton BF, Manns BJ, Zhang J, Tonelli M, Klarenbach S, Hemmelgarn BR: Impact of anemia on hospitalization and mortality in older adults. Blood. 2006, 107:3841-6. 10.1182/blood-2005-10-4308

8. Hong CH, Falvey C, Harris TB, et al.: Anemia and risk of dementia in older adults: findings from the Health ABC study. Neurology. 2013, 81:528-33. 10.1212/WNL.0b013e31829e701d

9. Chen-Edinboro LP, Murray-Kolb LE, Simonsick EM, Ferrucci L, Allen R, Payne ME, Spira AP: Association between non-iron-deficient anemia and insomnia symptoms in community-dwelling older adults: the Baltimore Longitudinal Study of Aging. J Gerontol A Biol Sci Med Sci. 2018, 73:380-5. 10.1093/gerona/glw332

10. Onder G, Penninx BW, Cesari M, et al.: Anemia is associated with depression in older adults: results from the InCHIANTI study. J Gerontol A Biol Sci Med Sci. 2005, 60:1168-72. 10.1093/gerona/60.9.1168

11. Penninx BW, Pahor M, Cesari M, et al.: Anemia is associated with disability and decreased physical performance and muscle strength in the elderly. J Am Geriatr Soc. 2004, 52:719-24. 10.1111/j.15325415.2004.52208.x

12. Balducci L: Anemia, fatigue and aging. Transfus Clin Biol. 2010, 17:375-81. 10.1016/j.tracli.2010.09.169

13. Chaves PH, Ashar B, Guralnik JM, Fried LP: Looking at the relationship between hemoglobin concentration and prevalent mobility difficulty in older women. Should the criteria currently used to define anemia in older people be reevaluated?. J Am Geriatr Soc. 2002, 50:1257-64. 10.1046/j.1532-5415.2002.50313.x

14. Riva E, Tettamanti M, Mosconi P, et al.: Association of mild anemia with hospitalization and mortality in the elderly: the Health and Anemia population-based study. Haematologica. 2009, 94:22-8. 
15. Penninx BW, Pahor M, Woodman RC, Guralnik JM: Anemia in old age is associated with increased mortality and hospitalization. J Gerontol A Biol Sci Med Sci. 2006, 61:474-9. 10.1093/gerona/61.5.474

16. den Elzen WP, Willems JM, Westendorp RG, de Craen AJ, Assendelft WJ, Gussekloo J: Effect of anemia and comorbidity on functional status and mortality in old age: results from the Leiden 85-plus Study. CMAJ. 2009, 181:151-7. 10.1503/cmaj.090040

17. Agnihotri P, Telfer M, Butt Z, et al.: Chronic anemia and fatigue in elderly patients: results of a randomized, double-blind, placebo-controlled, crossover exploratory study with epoetin alfa. J Am Geriatr Soc. 2007, 55:1557-65. 10.1111/j.1532-5415.2007.01357.x

18. Roy CN, Snyder PJ, Stephens-Shields AJ, et al.: Association of testosterone levels with anemia in older men: a controlled clinical trial. JAMA Intern Med. 2017, 177:480-90. 10.1001/jamainternmed.2016.9540

19. Shlush LI: Age-related clonal hematopoiesis. Blood. 2018, 131:496-504. 10.1182/blood-2017-07-746453

20. Gomes AC, Gomes MS: Hematopoietic niches, erythropoiesis and anemia of chronic infection . Exp Hematol. 2016, 44:85-91. 10.1016/j.exphem.2015.11.007

21. Makipour S, Kanapuru B, Ershler WB: Unexplained anemia in the elderly . Semin Hematol. 2008, 45:250-4. 10.1053/j.seminhematol.2008.06.003

22. Baylis D, Bartlett DB, Patel HP, Roberts HC: Understanding how we age: insights into inflammaging . Longev Healthspan. 2013, 2:8. 10.1186/2046-2395-2-8

23. Weiss G, Goodnough LT: Anemia of chronic disease. N Engl J Med. 2005, 352:1011-23. 10.1056/NEJMra041809

24. Wimazal F, Fonatsch C, Thalhammer R, et al.: Idiopathic cytopenia of undetermined significance (ICUS) versus low risk MDS: the diagnostic interface. Leuk Res. 2007, 31:1461-8. 10.1016/j.leukres.2007.03.015

25. Valent P: ICUS, IDUS, CHIP and CCUS: diagnostic criteria, separation from MDS and clinical implications . Pathobiology. 2019, 86:30-8. 10.1159/000489042

26. Valent P: Low erythropoietin production as non-oncogenic co-factor contributing to disease-manifestation in low-risk MDS: a hypothesis supported by unique case reports. Leuk Res. 2008, 32:1333-7. 10.1016/j.leukres.2008.02.022

27. Stauder R, Valent P, Theurl I: Anemia at older age: etiologies, clinical implications, and management . Blood. 2018, 131:505-14. 10.1182/blood-2017-07-746446

28. Tay HS, Soiza RL: Systematic review and meta-analysis: what is the evidence for oral iron supplementation in treating anaemia in elderly people?. Drugs Aging. 2015, 32:149-58. 10.1007/s40266-015-0241-5

29. Price E, Artz AS, Barnhart H, et al.: A prospective randomized wait list control trial of intravenous iron sucrose in older adults with unexplained anemia and serum ferritin 20-200 ng/mL. Blood Cells Mol Dis. 2014, 53:221-30. 10.1016/j.bcmd.2014.06.003 